\title{
Índice Prognóstico de Mortalidade BODE e Atividade Física em Doentes Pulmonares Obstrutivos Crônicos
}

\author{
BODE Prognostic Index of Mortality and Physical Activity in Chronic \\ Obstructive Pulmonary Patients
}

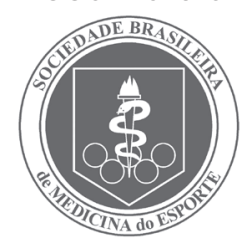

Artigo Original

\author{
Karen Muriel Simon ${ }^{1}$ \\ Ana Paula Hass² \\ Jorge Luiz Zimmermman ${ }^{3}$ \\ Marta Fioravanti Carpes ${ }^{4}$ \\ 1. Fisioterapeuta da Universidade \\ Tuiuti, Curitiba, PR. \\ 2. Fisioterapeuta da Universidade do \\ Vale do Itajaí-Univali, Itajaí, SC. \\ 3. Médico da Faculdade Evangélica \\ do Paraná, Curitiba,PR. \\ 4. Fisioterapeuta da Universidade \\ Federal de Santa Maria- UFSM , \\ Santa Maria, RS.
}

\section{Endereço para correspondência:} Karen Muriel Simon, Rua Nakle

Francisco, 343 - 88380-000

- Piçarras, SC.

E-mail: ksimon@univali.br

Submetido em 13/12/2007

Versão final recebida em 16/03/2008 Aceito em 27/05/2008

\begin{abstract}
RESUMO
Introdução e Objetivo: A DPOC é importante causa de mortalidade no mundo. Para predizer a mortalidade em pacientes com doença pulmonar obstrutiva crônica (DPOC), o índice BODE é utilizado e sua pontuação pode ser reduzida com a prática de atividade física. Este estudo objetiva verificar a associação entre o índice BODE com atividade física em indivíduos com DPOC. Métodos: Foi calculado o índice BODE usando as variáveis: distância no teste da caminhada de seis minutos (TC6'); Medical Research Council Dyspnea Score (MRC); índice de massa corpórea (IMC) e grau de obstrução ao fluxo aéreo $\left(V_{E F}\right)$. Os indivíduos foram divididos em grupos classificados em ativos fisicamente (AF) e inativos fisicamente (IF). O teste do qui-quadrado foi utilizado para testar a independência das variáveis. O teste $t$ foi utilizado para comparar as variáveis TC6, MRC, IMC, VEF 1 entre os grupos de indivíduos AF e IF. Resultados e Conclusão: Em 38 pacientes com moderada e grave obstrução ao fluxo aéreo $(36,5 \pm 11,9)$, o valor médio do BODE foi de 4,1 $\pm 2,1$ para o grupo de indivíduos AF; para o grupo de indivíduos $I F$, o valor médio do BODE foi de $6 \pm 2,2$. Houve diferença significativa na comparação das variáveis TC6,' MRC, BODE entre os grupos. Existiu maior ocorrência de indivíduos que praticam atividade física de se situar nos quartis um e dois e os indivíduos que não praticam atividade física se situarem nos quartis três e quatro. A existência da maior pontuação do BODE para os pacientes com DPOC considerados inativos fisicamente sustenta o conceito geral de que a inatividade aumenta o risco de morte.
\end{abstract}

Palavras-chave: doença obstrutiva, exercício, espirometria.

\begin{abstract}
Introduction: COPD is an important cause of morbidity and mortality in the world. The BODE is an important predictive index of mortality and its scores can be reduced with the practice of physical activity. Objective: To verify the association between BODE and physical activity in patients with chronic obstructive pulmonary disease (COPD). Methods: the BODE index was calculated using the following variables: distance in the 6-minute walk test (6MWT); Medical Research Council Dyspnea Score (MRC); body mass index (BMI) and degree of pulmonary function $\left(F E V_{1}\right)$. The patients were divided in groups classified as physically active (PA) or physically inactive (PI). Qui-square test was used to verify the independence of the variables. T-Student test was used to compare the 6WMT, MRC, BMI, FEV variables between PA and PI groups. Results and conclusion: In 38 patients with moderate and serious obstruction to the air flow ( $36.5 \pm 11.9)$, BODE mean value was of $4.1 \pm 2.1$ for the PA group, while for the PI group BODE mean value was of \pm 2.2 . Significant difference in the comparison of WD6, MRC, BODE variables between groups was observed. There was more occurrence of individuals who practice physical activity in quartiles one and two, while individuals who do not practice aerobic physical activity were in quartiles three and four. Higher scores of BODE for DPOC patients considered physically inactive supports the general concept that inactivity increases risk of death.
\end{abstract}

Keywords: obstructive disease, exercise, spirometry.

\section{INTRODUÇÃO}

A doença pulmonar obstrutiva crônica (DPOC) classifica-se atualmente como importante causa de morbidade e mortalidade no mundo. Caracteriza-se por obstrução parcialmente reversível da via aérea e resposta inflamatória pulmonar (efeitos pulmonares). Além disso, cursa com alterações nutricionais e da musculatura esquelética (efeitos extrapulmonares)(1).

Esse conjunto de alterações associado à hiperinsuflação dinâmica, que impede os pacientes de alcançar as demandas ventilatórias necessárias aos exercícios, favorece o surgimento da dispnéia ${ }^{(2,3)}$.
Geralmente, a dispnéia é observada durante a realização de atividades intensas, porém, para indivíduos com DPOC, essa é uma situação vivida em atividades do dia-a-dia ou até mesmo no repouso. A dispnéia é o principal agente causador do descondicionamento físico e este se deve principalmente à inatividade muscular. Os músculos em desuso tornam-se atrofiados, com redução do número de mitocôndrias e o número de enzimas oxidativas ${ }^{(2,4,5)}$.

A baixa capacidade ao exercício, a hiperinsuflação dinâmica, o aumento do grau de obstrução pulmonar, as alterações nutricionais, entre outros fatores, denotam a gravidade da doença ${ }^{(1)}$. 
Celli et al. (2004) criaram um índice preditor de mortalidade denominado BODE ( $B$ - body mass index; $O$ - airflow obstruction; $D$ - dyspnea; $E$ - exercise capacity) que avalia o grau de mortalidade dos indivíduos com DPOC de forma sistêmica. Esse índice não inclui somente a avaliação do grau de obstrução, mas também fatores como tolerância ao exercício, índice de massa corpórea e dispnéia dos indivíduos. Sua pontuação varia de zero a dez e, quanto maior a pontuação, maior o índice de mortalidade(6).

Pacientes com DPOC que não praticam o mínimo de atividade física diária acrescem o grau de mortalidade. Pitta et al. (2006) verificaram, em pacientes gravemente comprometidos pela doença, aumento significativo do BODE para cada dia de inatividade física $^{(7)}$.

É considerado fisicamente ativo o indivíduo que pratica diariamente atividade de níveis moderados e intensos, como caminhadas por um período de 30 minutos, três vezes por semana ou preferencialmente todos os dias da semana (Guidelines of the American College of Sports Medicine (ACSM, 1995) (8)

Assim, este estudo teve como objetivo verificar a associação entre o índice preditor de mortalidade BODE e suas variáveis com atividade física em pacientes com doença pulmonar obstrutiva crônica moderada e grave.

\section{MÉTODOS}

Trinta e oito indivíduos com diagnóstico de DPOC moderada e grave participaram do estudo. Critérios de inclusão: Diagnóstico de DPOC $\left(\mathrm{VEF}_{1} / \mathrm{CVF}<70 \%\right.$ e VEF1 < 70\% do valor previsto) ${ }^{(1)}$, história de tabagismo acima de 20 anos/maço, estabilidade clínica no último mês. Critérios de exclusão: Incapacidade de executar qualquer uma das avaliações do estudo, exacerbação da doença durante o período da coleta, presença de doenças associadas como: miocardiopatias, doenças musculoesqueléticas, tuberculose e asma.

Todos os indivíduos assinaram o termo de consentimento livre e esclarecido, sendo o estudo aprovado pelo Comitê de Ética em Pesquisa em Humanos da Universidade do Vale do Itajaí.

Inicialmente, os voluntários foram pesados e medidos, depois realizaram espirometria pré e pós- broncodilatador $(\mathrm{BD})^{(9)}$, dois testes da caminhada de seis minutos (TC6 $)^{\prime(10)}$ e a seguir foram aplicados a escala de dispnéia Medical Research Council (MRC) ${ }^{(11)}$ e questionados quanto à realização ou não de atividade física.

Os indivíduos foram considerados ativos fisicamente quando praticavam atividade física de nível moderado e intenso, por um período de 30 minutos todos os dias ou três dias semanais ${ }^{(8)}$.

A partir dos resultados do IMC, grau de obstrução, distância percorrida no TC6', dispnéia, foi calculado o índice preditor de mortalidade $B O D E$, dividido nos quartis: um (pontuação de zero a dois), dois (três a quatro), três (cinco a seis) e quatro (sete a 10).

$\mathrm{Na}$ análise estatística foi aplicado o teste de normalidade de Kolmogorov-Smirnov nas variáveis. Os dados foram descritos como média, desvio padrão e mediana.

Foi aplicado o teste $t$ de Student para comparar o BODE e suas variáveis IMC, VEF 1 , MRC e TC6' com o grupo dos pacientes ativos fisicamente e o grupo de pacientes inativos fisicamente. $O$ teste do qui-quadrado foi utilizado para estabelecer a independência entre atividade física e o índice preditor de mortalidade BODE.

O nível de significância adotado para o tratamento estatístico foi de $5 \%(p<0,05)$.

\section{RESULTADOS}

A amostra foi composta por 38 pacientes, destes, 27 (71\%) eram do sexo masculino e apresentavam moderado grau de obstrução pulmonar de acordo a classificação do Gold ${ }^{(1)}$
Do número total de pacientes, 15 (39\%) foram considerados fisicamente ativos e 23 (61\%), fisicamente inativos, segundo critérios do American College of Sports Medicine (ACSM) ${ }^{(8)}$.

As características dos pacientes estão apresentadas na tabela 1.

Tabela 1. Médias, desvio padrão, mediana das variáveis: idade; anos/maço; BODE; IMC; VEF 1 \%previsto; MRC e distância TC $6^{\prime}$ dos indivíduos fisicamente ativos e fisicamente inativos

\begin{tabular}{|c|c|c|c|c|c|c|}
\hline & \multicolumn{3}{|c|}{$\begin{array}{l}\text { Fisicamente ativos } \\
\qquad(n=15)\end{array}$} & \multicolumn{3}{|c|}{$\begin{array}{l}\text { Fisicamente inativos } \\
\qquad(\mathrm{n}=23)\end{array}$} \\
\hline & Média & $\mathrm{DP}$ & Mediana & Média & DP & Mediana \\
\hline Idade (anos) & 67 & 6 & 69 & 65 & 9 & 66 \\
\hline Anos/maço & 49,3 & 26,4 & 40,0 & 47,7 & 21,7 & 36 \\
\hline BODE & 4,1 & 2,1 & 3,0 & 6 & 2,2 & 6 \\
\hline IMC $\left(\mathrm{kg} / \mathrm{m}^{2}\right)$ & 24,5 & 4,2 & 24,6 & 23,5 & 4,4 & 21,7 \\
\hline $\mathrm{VEF}_{1} \%$ prev & 40,7 & 11,7 & 42,2 & 33,7 & 11,4 & 32,4 \\
\hline MRC & 2,3 & 1 & 2,0 & 3 & 0,9 & 3 \\
\hline $\mathrm{TC}^{\prime}(\mathrm{m})$ & 401,3 & 130,5 & 417 & 289,2 & 121,5 & 294 \\
\hline
\end{tabular}

DP: desvio padrão; BODE: índice preditor de mortalidade; $V E F_{1} \%$ previsto: grau de obstrução ao fluxo aéreo; IMC: índice de massa corporal; TC6': teste de caminhada de seis minutos; MRC: Medical Research Council.

O número total de indivíduos que praticam ou não atividade física foram agrupados nos quartis do índice BODE, sendo três ativos e um inativo no quartil um, seis ativos e cinco inativos no quartil dois, quatro ativos e sete inativos no quartil três e dois ativos e 10 inativos no quartil quatro. Os pacientes que obtiveram o resultado no BODE aproximando-se da pontuação 10 apresentaram pior prognóstico, ou seja, maior grau de mortalidade.

O estudo não mostrou diferença significativa entre os grupos ativos fisicamente e inativos fisicamente para as variáveis: idade; índice de massa corpórea; anos/maço e VEF 1 . Entretanto, houve diferença significativa para as variáveis: distância percorrida no TC6,' dispnéia pela escala MRC e o índice BODE entre os dois grupos (gráficos 1, 2 e 3).

Foi testada a independência entre a atividade física e o índice preditor de mortalidade BODE (tabela 2).

Houve maior ocorrência de indivíduos que praticam atividade física de se situarem nos quartis um e dois e os indivíduos que não praticam atividade física aeróbia, nos quartis três e quatro.

\section{Teste da caminhada de seis minutos}

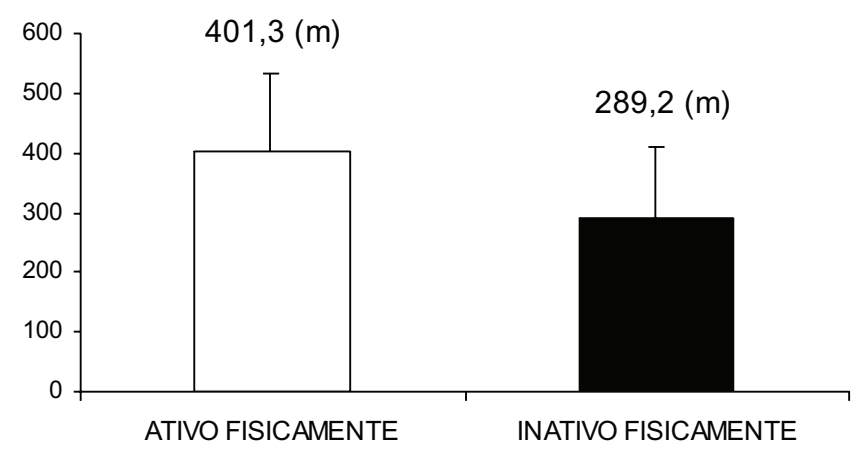

Gráfico 1. Médias \pm desvios padrão da distância percorrida no teste da caminhada de seis minutos dos indivíduos fisicamente ativos e fisicamente inativos. ${ }^{*} p<0,05$ 


\section{Índice preditor de mortalidade BODE}

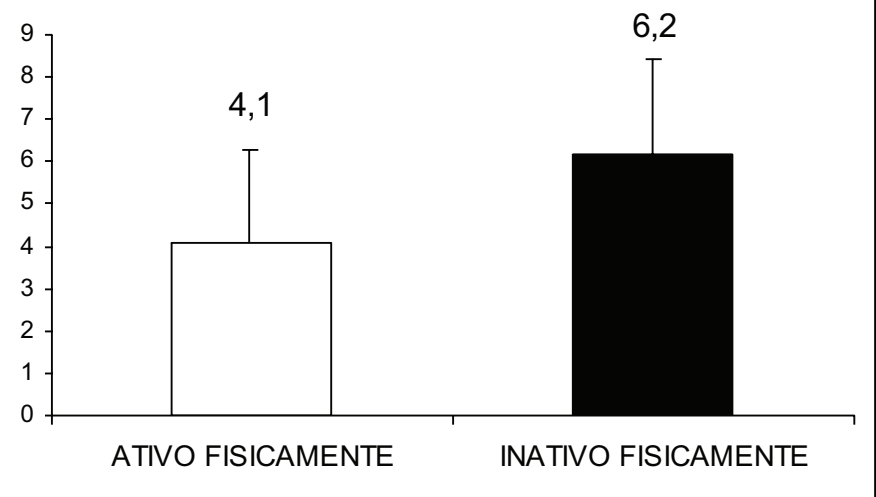

Gráfico 2. Médias \pm desvios padrão do índice preditor de mortalidade BODE dos indivíduos fisicamente ativos e fisicamente inativos.

${ }^{*} p<0,05$

\section{Escala de Dispnéia (MRC)}

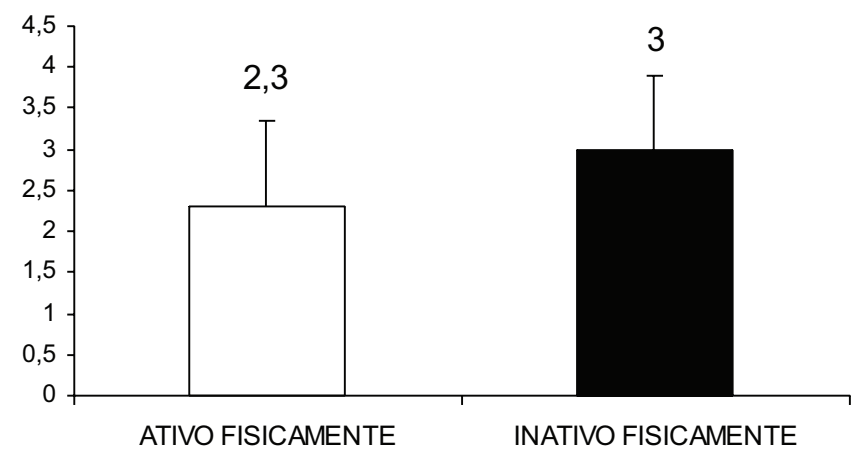

Gráfico 3. Médias \pm desvios padrão da escala MRC para os indivíduos fisicamente ativos e fisicamente inativos.

${ }^{*} p<0,05$.

Tabela 2. Análise de freqüência cruzada, BODE e atividade física

\begin{tabular}{c|c|c|c}
\hline BODE & \multicolumn{3}{|c}{ Atividade física } \\
\hline Freqüência \% & Sim & Não & Total \\
\hline$Q_{1}+Q_{2}$ & 9 & 6 & 15 \\
\hline$Q_{3}+Q_{4}$ & 6 & 17 & 23 \\
\hline Total & 15 & 23 & 38 \\
\hline
\end{tabular}

O valor do qui-quadrado calculado foi de 4,37, $\operatorname{com} p=0,036$.

A hipótese de independência foi rejeitada com probabilidade de erro ( $p=0,036)$. Assim sendo, pode-se afirmar com $97 \%$ de confiabilidade que a atividade física está associada com o escore do índice preditor de mortalidade BODE.

\section{DISCUSSÃO}

O presente estudo teve por finalidade verificar a existência de associação entre o índice preditor de mortalidade BODE com atividade física em pacientes com moderada a grave doença pulmonar obstrutiva crônica (DPOC).

Dos 38 indivíduos avaliados, 15 indivíduos eram ativos fisicamente (AF) e 23 inativos fisicamente (IF). De acordo com Pate et al. (1995), é recomendado um mínimo de 30 minutos diários de atividade física de moderada intensidade (caminhada) para aumentar o índice de sobrevida em indivíduos saudáveis ${ }^{(8)}$.

Comparando o índice preditor de mortalidade BODE do grupo de indivíduos classificados em AF com os indivíduos IF, verificou-se maior índice de mortalidade para os indivíduos IF. Segundo ACSM, indivíduos com DPOC considerados inativos fisicamente apresentam maior índice de mortalidade verificado pelo $B O D E^{(7)}$.

Entre as variáveis classificadas pelo BODE não se observou diferença significativa entre os grupos IF e AF para o IMC e VEF, . Enquanto para as variáveis: distância percorrida no TC6'e escala de dispnéia MRC, essa situação foi observada.

A variável fisiológica $V E F_{1}$ está intimamente relacionada ao grau de mortalidade da DPOC. Pacientes que possuem grau de obstrução pulmonar elevado podem limitar as atividades físicas, porém a atividade física não exerce influência na variável VEF, dos indivíduos com DPOC (12-14)

Esse fato concorda com a literatura pertinente, reafirmando que a prática de exercícios aeróbios em programas de reabilitação não influenciam no grau de obstrução pulmonar e, sim, nos distúrbios secundários à DPOC (15). Embora, Casaburi et al. (1997) em seu trabalho tenham verificado redução significativa do grau de obstrução pulmonar em indivíduos com grave DPOC após a participação de programas de reabilitação, esse não é um resultado comumente observado após a prática de atividade física(16).

O elevado grau de obstrução pulmonar promove o surgimento da dispnéia, que é um fator importante na limitação do exercício(17). Essa variável também é importante preditor de mortalidade na DPOC ${ }^{(14)}$.

Na DPOC freqüentemente os indivíduos encontram-se limitados na realização das atividades físicas. Essa limitação pode acontecer devido à presença de inúmeros fatores, como a progressiva obstrução pulmonar ${ }^{(3)}, \mathrm{o}$ aumento da dispnéia ${ }^{(18)}$, a hiperinsuflação dinâmica(2), fraqueza muscular periférica ${ }^{(19)}$, fatores que explicam a interferência da atividade física na redução da dispnéia e no aumento da distância percorrida no TC6' no grupo AF.

Categorizar o paciente DPOC baseado em níveis de dispnéia mostra correlação mais forte em relação à sobrevida quando comparado com a classificação da severidade da doença acessada pelo $\mathrm{VEF}_{1}{ }^{(18)}$.

A dispnéia, verificada pela escala MRC, mostrou maior grau de comprometimento para o grupo de pacientes IF quando comparado com o grupo de pacientes AF. A mediana da escala MRC observada nos grupos foi de dois para o grupo que praticava atividade física e três para o grupo que não praticava atividade física. $\mathrm{O}$ grupo inativo classificou-se como gravemente comprometido pela sensação de dispnéia em atividades de diferente intensidade.

A atividade física, como programas de reabilitação pulmonar, é capaz de reduzir o grau de dispnéia dos indivíduos com DPOC (20).

Comparando a variável IMC, não houve diferença significativa entre os grupos AF e IF. Tal fato pode ter ocorrido devido à baixa variabilidade dos valores desse índice entre os dois grupos analisados.

O papel da atividade física no controle do peso corporal e da massa de gordura tem sido amplamente estudado; existe uma correlação negativa entre a atividade física regular e o IMC (21). Alguns autores afirmam que há relação entre o baixo IMC com o comprometimento muscular periférico e conseqüentemente com a baixa capacidade ao exercício em indivíduos com DPOC (22-26). Porém, para a opinião de outros autores, O IMC pode não identificar adequadamente a perda da massa muscular em pacientes com DPOC ${ }^{(19,27)}$.

Quando comparado o TC6' entre os grupos AF e IF, foi observada distância percorrida significantemente inferior para o grupo de pa- 
cientes IF. A baixa distância percorrida no teste da caminhada de seis minutos para pacientes DPOC marca a inatividade física e é capaz de predizer maior índice de mortalidade ${ }^{(7,28)}$.

Com a inatividade muscular, os músculos dos membros inferiores atrofiam-se, a densidade capilar muscular e a concentração de enzimas oxidativas reduzem-se e as fibras musculares alteram-se em sua constituição. Existe desequilíbrio entre a oferta e demanda de oxigênio que determinam a redução do metabolismo aeróbio muscular, com conseqüente perda de resistência e força muscular ${ }^{(4)}$. Pacientes com DPOC permanecem significantemente menor parte do tempo caminhando e maior parte do tempo na posição deitada e sentada quando comparados com indivíduos saudáveis ${ }^{(28)}$.

A inatividade física em pacientes DPOC acarreta piora da capacidade funcional verificado pelo TC6, piora do grau de dispnéia constatado pela escala MRC e piora do índice preditor de mortalidade $B O D E$, indicando elevado índice de mortalidade para esta população(7).

Visto que pacientes com DPOC encontram-se limitados em suas atividades e, quanto maior a gravidade da doença, maior a limitação para atividades de vida diária(29) e maior o risco de morbidade e mortalidade, assim, conhecendo os benefícios que o exercício proporciona para os indivíduos com DPOC, torna-se essencial a inserção destes na prática de atividade física, visando redução da morbidade e mortalidade.

Pitta et al. ${ }^{(7)}$ observaram em indivíduos com DPOC a relação da inatividade física com o maior índice de mortalidade verificado pelo BODE.

Neste estudo, a existência da maior pontuação do BODE para os pacientes com DPOC, considerados inativos fisicamente, sugere o conceito de que a inatividade pode aumentar o risco de morte.

Uma possível limitação observada neste estudo foi o fato de que os indivíduos da amostra foram questionados quanto à realização ou não de atividade física, não havendo acompanhamento prospectivo para confirmar os achados.

Observou-se também que a variável IMC utilizada no índice BODE como um dos fatores preditores de mortalidade não verifica apenas a perda da massa magra dos indivíduos e talvez isso possa ter contribuído para ocultar o real comprometimento muscular ocasionado pela doença pulmonar obstrutiva.

Todos os autores declararam não haver qualquer potencial conflito de interesses referente a este artigo.

\section{REFERÊNCIAS BIBLIOGRÁFICAS}

1. Global Initiative for Chronic Obstructive Lung Disease. Global Strategy for the Diagnosis, Management and Prevention of Chronic Obstructive Pulmonary Disease; Disponível em URL: http://www.goldcopd.org.

2. Marin J, Carrizo SJ, Gascon M, Sánchez A, Gallego B, Celli BR. Inspiratory capacity, dynamic hyperinflation, breathlessness, and exercise performance during the six minute walk test in chronic obstructive pulmonary disease. Am J Respir Crit Care Med. 2001;163(6):1395-9.

3. Reid RS, Días OP, Jorquera A, Lisboa CB. Efecto de la prueba de la caminata de seis minutos sobre la hiperinflación pulmonar en pacientes con enfermidad pulmonar obstructiva crónica avanzada. Rev Med Chile. 2001;129(10):1171-8.

4. Casaburi R. Skeletal muscle function in COPD. Chest. 2000;117(5 suppl 1):S267-71.

5. Donaldson G, Seemungal T, Patel I, Browmik A, Wilkinson TMA, Hurst JR, et al. Airway and systemic inflammation and decline in lung function in patients with COPD. Chest. 2005;128(1):1995-2004.

6. Celli RB, Cote CG, Marin JM, Casanova C, Oca MM, Mendez RA, et al. The body-mass index, airflow, obstruction, dyspnea and exercise capacity index in chronic obstructive pulmonary disease. N Eng J Med. 2004;350(10):1005-12.

7. Pitta F, Troosters T, Probst VS, Lucas S, Decramer M, Gosselink R. Potential consequences for stable chronic obstructive pulmonary disease patients who do not get the recommended minimum daily amount of physical activity. J Bras Pneumol. 2006;32(4):301-8.

8. Pate RR, Pratt M, Blair SN, Haskell WL, Macera CA, Bouchard C, et al. Physical activity and public health. A recommendation from the Centers for Disease Control and Prevention and the American College of Sports Medicine. JAMA. 1995;273(5):402-7.

9. American Thoracic Society. Standards for the diagnosis and care of patients with chronic obstructive pulmonary disease. Am J Respir Crit Care Med. 1995;152(1):1107-36.

10. American Thoracic Society. Statement: Guideline for the six-minute walk test. Am J Respir Crit Care Med. 2002;166(1):111-7.

11. Mahler D, Wells C. Evaluation of clinical methods for rating dyspnea. Chest. 1998;93(3):580-6.

12. Babb TG, Viggiano B, Hurley B, Staats B, Rodarte JR. Effect of mild-to-moderate airflow limitation on exercise capacity. Am Physiol Society. 1990;91(1):223-31.

13. Oca MM, Rassulo J, Celli BR. Respiratory muscle and cardiopulmonary function during exercise in very severe COPD. Am J Respir Crit Care Med. 1996;154(5):1284-9.

14. Oga T, Nishimura K, Tsukino M, Sato S, Hajiro T. Analysis of the factors related to mortality in chronic obstructive pulmonary disease. Am J Respir Crit Care Med. 2003;167(4):544-9.

15. Garrod R, Bestall JC, Paul EA, Wedzicha JA, Jones PW. Development and validation of standardized measure of activity of daily living in patients with severe COPD: the London Chest Activity of Daily Living Scale (LCADL). Respir Med. 2000;94(6):589-96.
16. Casaburi R, Porszasz J, Burns MR, Carithers ER, Chang RS, Cooper CB. Physiologic benefits of exercise training in rehabilitation of patients with severe chronic obstructive pulmonary disease. Am J Respir Crit Care Med. 1997;155(5):1541-55.

17. Velloso M, Stella SG, Cendon S, Silva AC, Jardim JR. Metabolic and ventilatory parameters of four activities of daily living accomplished with arms in COPD. Chest. 2003;123(4):1047-53.

18. Hajiro T, Nishimura K, Tsukino M, Ikeda A, Oga T, Izumi T. A comparison o the level of dyspnea vs. disease severity in indicating the health- related quality of life of patients with COPD. Chest 1999;116(6):1632-7.

19. Belman MJ. Exercise in patients with chronic obstructive pulmonary disease. Thorax 1993;48(1):936-46.

20. McArdle W, Katch F, Katch V. Fisiologia do exercício: Energia, nutrição e desempenho humano. $4^{a}$ ed São Paulo: Guanabara Koogan; 1998.

21. Gosselink R, Troosters T, Decramer M. Peripheral muscle weakness contributes to exercise limitation in COPD. Am J Respir Crit Care Med. 1996;153(3):976-80.

22. Kobayashi A, Yoneda T, Yoshikawa M, Ikuno M, Takenaka H, Fukuoka A, et al. The relation of fat-free mass to maximum exercise performance in patients with chronic obstructive pulmonary disease. Springer New York. 2000;178(2):119-27.

23. Yoshikawa M, Yoneda T, Takenaka H, Fukuoka A, Okamoto Y, Narita N. Distribution of muscle mas and maximal exercise performance in patients with COPD. Chest. 2001;119(1):93-8.

24. Debigaré R, Marquis k, Côté C, Tremblay RR, Michaud A, Leblanc P. Catabolic/anabolic balance and muscle wasting in patients with COPD. Chest. 2003;124(1):83-9.

25. Saey D, Debigaré R, Leblanc $P$, Mador MJ, Côté $C H$, Jobin J, et al. Contractile leg fatigue after cycle exercise: a fator limiting exercise in patients with chronic obstructive pulmonary disease. Am J Respir Crit Care Med. 2003;168(4):425-30.

26. Eid AA, Ionescu AA, Nixon L, Lewis-Jenkins V, Matthews SB, Griffiths TL, et al. Inflamatory response and body composition in chronic obstructive pulmonary disease. Am J Respir Crit Care Med. 2001;164(8):1414-8

27. Mador MJ, Kufel TJ, Pineda L. Quadriceps fatigue after cycle Exercise in patients with Chronic Obstructive Pulmonary disease. Am J Respir Crit Care Med. 2000;161(2)447-53.

28. Pitta F, Troosters T, Spruit MA, Decramer PM, Gosselink R. Characteristics of physical activities in daily life in chronic obstructive pulmonary disease. Am J Respir Crit Care Med. 2005;171(9):972-7.

29. Garrod R, Paul EA, Wedzicha JA. An evaluation of the reliability and sensitivity of the London Chest Activity of Daily Living Scale (LCADL). Respir Med. 2002;96(9):725-30. 